\title{
Transcriptome and phytohormone analysis reveals a comprehensive phytohormone and pathogen defence response in pear self-/cross-pollination
}

\author{
Dongqing Shi ${ }^{1}$ - Chao Tang ${ }^{1} \cdot \operatorname{Runze} \mathrm{Wang}^{1} \cdot \mathrm{Chao} \mathrm{Gu}^{1} \cdot \mathrm{Xiao}^{\mathrm{Wu}}{ }^{1}$. \\ Shi $\mathrm{Hu}^{1} \cdot$ Jin Jiao ${ }^{1} \cdot$ Shaoling Zhang ${ }^{1}$
}

Received: 21 April 2017/ Accepted: 5 August 2017/Published online: 8 September 2017

(c) The Author(s) 2017. This article is an open access publication

\begin{abstract}
Key message Candidate genes were identified and the role of phytohormones such as JA-Me and ABA in the synthesis of S-RNase was emphasized in pear selfincompatibility.

Abstract Self-incompatibility (SI) occurs widely in flowering plants as an intraspecific reproductive barrier. This phenomenon promotes variation within species, but for some species such as Pyrus, SI is a nuisance rather than a benefit in agricultural production. Although many studies
\end{abstract}

Communicated by Ray J. Rose.

Electronic supplementary material The online version of this article (doi:10.1007/s00299-017-2194-0) contains supplementary material, which is available to authorized users.

Shaoling Zhang

slzhang@njau.edu.cn

Dongqing Shi

shidongqing@aliyun.com

Chao Tang

2015204013@njau.edu.cn

Runze Wang

rwang0901@gmail.com

Chao Gu

guchao@niau.edu.cn

Xiao Wu

wu_xiao299@163.com

Shi $\mathrm{Hu}$

2015104047@njau.edu.cn

Jin Jiao

2014104046@njau.edu.cn

1 Centre of Pear Engineering Technology Research, State Key Laboratory of Crop Genetics and Germplasm Enhancement, Nanjing Agricultural University, Nanjing 210095, China have been conducted on SI in pears, its mechanism remains unclear. In this study, high-throughput Illumina RNA sequencing (RNA-seq) was used to identify SI-related genes in pear styles. Using transcriptome comparisons, differentially expressed genes of unpollinated (UP), crosspollinated (CP), and self-pollinated (SP) styles were identified after $48 \mathrm{~h}$. A total of 1796 and 1890 genes were identified in DSC (UP vs. CP) and DSI (UP vs. SP), respectively. KEGG analysis revealed that genes involved in the "plant hormone signal transduction pathway" and "plant-pathogen interaction pathway" were significantly enriched in DSI (UP vs. SP) compared to those in DSC (UP vs. CP). The expression level of S-glycoprotein ribonuclease (S-RNase) was dramatically reduced in cross-pollinated $(\mathrm{CP})$ styles. To better understand the relationship between the expression patterns of S-RNase and two major KEGG pathways, the concentrations of phytohormones were measured, and the expression pattern of S-RNase was analysed using qRT-PCR. Our results demonstrate that methyl jasmonate and abscisic acid may enhance the expression level of S-RNase, and pollination can affect the synthesis of methyl jasmonate and abscisic acid in pear styles. Overall, this study is a global transcriptome analysis of SI in pear. A relationship between self-rejection, plant hormones, and pathogen defence was shown in pear.

Keywords Pear (Pyrus bretschneideri Rehd.) · Selfincompatibility $\cdot$ Transcriptome $\cdot$ S-RNase $\cdot$ Methyl jasmonate
Abbreviations
24 HAP 24 Hours after pollination
48 HAP 48 Hours after pollination
AMPK 5'-AMP-activated protein kinase
NCED 9-cis-epoxycarotenoid dioxygenase 


$\begin{array}{ll}\text { AOC } & \text { Allene-oxide cyclase } \\ \text { AIPT } & \text { Adenylate isopentenyltransferase } \\ \text { ABA } & \text { Abscisic acid } \\ \text { BR } & \text { Brassinolide } \\ \text { BRI } & \text { Brassinosteroid insensitive } \\ \text { CK } & \text { Cytokinin } \\ \text { CYCD } & \text { Cyclin D } \\ \text { CP } & \text { Cross-pollinated } \\ \text { DEGs } & \text { Differentially expressed genes } \\ \text { UP vs. } & \text { DSC } \\ \text { CP } & \\ \text { UP vs. } & \text { DSI } \\ \text { SP } & \\ \text { FDR } & \text { False discovery rate } \\ \text { GSI } & \text { Gametophytic SI } \\ \text { GA3 } & \text { Gibberellins } \\ \text { GID } & \text { GA insensitive dwarf } \\ \text { DELLA } & \text { GA sensing } \\ \text { AHK } & \text { Histidine kinase } \\ \text { ATHB } & \text { Homeobox-leucine zipper protein ATHB } \\ \text { RNA-seq } & \text { High-throughput Illumina RNA sequencing } \\ \text { IAA } & \text { Indole-3-acetic acid } \\ \text { MYC } & \text { Jamonate associated MYC } \\ \text { KEGG } & \text { Kyoto Encyclopedia of Genes and Genomes } \\ \text { FLS } & \text { Leucine-rich-repeat receptor kinases flagellin } \\ & \text { sensing } \\ \text { JA-Me } & \text { Methyl jasmonate } \\ \text { PPL } & \text { Pectate lyase } \\ \text { PCD } & \text { Programmed cell death } \\ \text { EFR } & \text { Receptor-like serine/threonine-protein kinase } \\ \text { GABA } & \gamma \text {-Amino butyric acid } \\ \text { SP } & \text { Self-pollinated } \\ \text { CTR } & \text { Serine/threonine-protein kinase } \\ \text { S-RNase } & \text { S-glycoprotein ribonuclease } \\ \text { SSI } & \text { Sporophytic SI } \\ \text { SI } & \text { Self-incompatibility } \\ \text { WRKY } & \text { Transcription factor WRKY } \\ \text { PCL } & \text { Transcription factor PCL } \\ \text { TTS } & \text { Transmitting tissue-specific proteins } \\ \text { UP } & \text { Unpollinated } \\ \text { UBEH } & \text { Ubiquitin-conjugating enzyme E2 H } \\ \text { UBEM } & \text { Ubiquitin-conjugating enzyme E2 M } \\ \text { CP vs. } & \text { X } \\ \text { SP } & \\ \text { ZAT } & \text { Zinc finger protein } \\ \text { ZR } & \text { Zeatin riboside } \\ & \end{array}$

\section{Introduction}

Most flowering plants with hermaphrodite flowers have evolved a strategy to prevent self-pollination and promote outcrossing. A ubiquitous strategy is self-incompatibility
(SI), which inhibits the growth of self-pollen and accepts the non-self for fertilization (de Nettancourt 1997). Decades of research in biochemistry and molecular biology have gradually uncovered the mechanism of SI (McClure 2004; Nasrallah and Nasrallah 2014; Wilkins et al. 2015). However, the mechanism of pollen tube growth and style recognition remain unclear.

There are two major types of SI: sporophytic SI (SSI), found in Brassicaceae, and gametophytic SI (GSI), found in Rosaceae and Solanaceae. SSI is controlled by the interaction between stigma-specific S-receptor kinase and a pollen-specific S-locus cysteine-rich protein, which inhibits self-pollen only on the surface of the stigma (Indriolo et al. 2014). By contrast, during GSI in Rosaceae and Solanaceae, the pollen tube penetrates into the base of the style, and S-glycoprotein ribonuclease (S-RNase) activity and the pollen-specific S-locus F-box protein (SLF/SFB) determine cross-fertilization and self-inhibition of growth (Liu et al. 2014). Furthermore, pollen-pistil recognition begins in the stigma. This reaction continues until the pollen tube reaches the base of the style in most GSI systems. The GSI mechanism in Papaveraceae involves style-specific ligands that bind to receptors on the membrane of the incompatible pollen tube and trigger a $\mathrm{Ca}^{2+}$ signalling cascade, which results in programmed cell death (PCD) of the self-pollen (Takayama and Isogai 2005). Different SI types exhibit different biological processes in space and time.

Pollen tube growth in the style is hyponastic growth during which there is a continuous exchange of signals between the pollen tube and style. RNA degradation is triggered, and a signalling cascade results in PCD culminated by factors that determine style incompatibility (Eaves et al. 2014; Wilkins et al. 2014). Proteins that contribute to reinforcement and secreted proteins, including potential signalling components, are significantly enriched during pollination (Tung et al. 2005). Stigma/stylar cysteine-rich adhesion proteins, chemocyanin, transmitting tissue-specific proteins (TTS), and $\gamma$-amino butyric acid (GABA) have been identified in directing pollen tube growth (Swanson et al. 2004). Intriguingly, TTS binds to S-RNase in Nicotiana (Hancock et al. 2003). In GSI, the compatible pollen tube in the style sends a signal to reduce the S-RNase level (Liu et al. 2009), which suggests reciprocal signalling crosstalk between the pollen tube and style cells of the transmitting tract.

Overall, SI is a biological process that is controlled by multiple genes and a reciprocal interaction between pollen and the style. High-throughput Illumina RNA sequencing (RNA-seq) is a powerful method to investigate gene regulation and the molecular basis of SI. Candidate genes contributing to SI interaction have been successfully identified in Leymus chinensis (Zhou et al. 2014), Citrus 
limon (Zhang et al. 2015), Solanum (Zhao et al. 2015), and Camellia sinensis (Zhang et al. 2016).

Pear, a perennial and self-incompatible species in the Rosaceae family, is an ancient fruit crop that is cultivated worldwide (Wu et al. 2013). There are large-scale plantings of pear in China. Owing to the lack of self-pollination, considerable time and effort are required every year for the collection of large amounts of pollen and the pollination of trees using pollen consisting of different $\mathrm{S}$ alleles. This results in an obvious wastage of land and is not only timeconsuming but also causes problems in managing different cultivars. It would be beneficial to farmers if SI could be prevented. S-RNase, is a basic protein with a PI of 9.3-10.0 and a molecular mass of $30 \mathrm{kDa}$, plays a key role in the inhibition of self-pollen through RNA degradation activity (Hiratsuka et al. 2001). Several studies have been conducted to clarify the mechanism of S-RNase cytotoxicity in incompatible pollen in pear. For example, S-RNase has been reported to trigger mitochondrial alteration and to decrease NADPH oxidase activity in the mitochondria and cytosol (Wang et al. 2010). As a result, tip-localized ROS were disrupted, the nuclear DNA was degraded (Wang et al. 2009), the balance of intracellular $\mathrm{Ca}^{2+}$ was altered, and the stability of the actin cytoskeleton was broken (Liu et al. 2007). However, a little work has been conducted on the synthesis and regulation of S-RNase in pear styles. S-RNase activity is necessary for the rejection of incompatible pollen (Huang et al. 1994), and a minimum concentration of S-RNase is also required in the style (Qin et al. 2006). Thus, inhibition of S-RNase activity or a reduction in S-RNase levels in styles may be a good method to prevent the rejection of self-pollen.

In this study, RNA-seq was used to reveal the gene regulation of self- and cross-pollination in pear to better understand SI. Phytohormone analysis and phytohormone treatment combined with RT-PCR were used to establish the relationship between phytohormones and SI in Pyrus.

\section{Materials and methods}

\section{Plant material}

Adult pear trees were planted in the orchards of Nanjing Agricultural University, Jangsu, China. The anthers were detached from 'Dangshan' pear trees before anthesis. Then, the styles were pollinated with self-pollen ('Dangshan') and cross-pollen ('Cuiguan'). Pollination bags were used to ensure that the styles were not subjected to other types of interference. After 24 and $48 \mathrm{~h}$ pollination, unpollinated, self-pollinated, and cross-pollinated styles with stigma (containing pollen or not) were collected, immediately frozen in liquid nitrogen, and stored at $-80{ }^{\circ} \mathrm{C}$.
Anthers were detached first; then, the styles were soaked in JA-Me (Sigma, St. Louis, MO, USA), ABA (RYON, Shanghai, China), GA3 (Genview, IL, USA), IAA (Genview, IL, USA), BR (Sigma, St. Louis, MO, USA), and ZR (COMIN, Shanghai, China) for $3 \mathrm{~min}$ at the final concentrations of $10,50,50,200,5$, and $100 \mu \mathrm{M}$, respectively. Subsequently, pollination bags were used to ensure that the styles were not subjected to other types of interference. All hormones were added to $1 \%$ ethanol containing $0.1 \%$ Tween 20 solution. This solution was used as the mock control. After $0,4,12,24$, and $48 \mathrm{~h}$ exogenous treatment, UP styles with stigma were collected, immediately frozen in liquid nitrogen, and stored at $-80{ }^{\circ} \mathrm{C}$.

\section{RNA isolation and Illumina sequencing}

The RNAprep Pure Plant Kit (Tiangen, Beijing, China) was used to isolate total RNA according to the manufacturer's protocol. RNA degradation and contamination were detected on $1 \%$ agarose gels. The RNA concentration of these samples was measured using a NanoDrop 2000 (Thermo Scientific, Waltham, MA, USA). The quality was assessed using an Agilent 2100 Bioanalyzer (Agilent Technologies, CA, USA). Only samples with RIN (RNA integrity number) $\geq 8$ and $28 \mathrm{~S}: 18 \mathrm{SRNA} \geq 1.5$ were used for deep sequencing. The cDNA libraries were prepared from different style samples after $48 \mathrm{~h}$ using a TruSeq ${ }^{\mathrm{TM}}$ RNA Sample Prep Kit (Illumina, Carlsbad, CA, USA) following the manufacturer's protocol. Briefly, poly(A) mRNA was enriched using poly-T oligoattached magnetic beads (Invitrogen, Foster City, USA) and was then fragmented into short pieces using an RNA Fragmentation Kit (Ambion, Austin, TX, USA). Double-stranded cDNA was reverse transcribed and amplified. An Illumina HiSeq ${ }^{\mathrm{TM}} 4000$ instrument was used to perform the sequencing reactions.

\section{HiSeq data analysis}

Raw reads containing by the HT-2500 were pass-filtered to exclude reads that contain adapters, low-quality, and unknown bases. The remaining high-quality sequences (clean reads) were mapped to the Pyrus bretschneideri genome data (Wu et al. 2013) (http://www.peargenome. njau.edu.cn) using Tophat (Trapnell et al. 2012), permitting no more than five base mismatches in the alignment. The mapped reads were counted using HT-Seq (Anders et al. 2014).

\section{Identification of DEGs}

Quantification of transcript expression was performed using the Reads Per kb per Million reads (RPKM) method (Mortazavi et al. 2008). Differentially expressed genes 
(DEGs) analysis was performed using the method described by Audic (Audic and Claverie 1997). False discovery rate (FDR) was used to determine the $P$ value thresholds in multiple testing. In the statistical analysis, $P$ values of $<0.05$ and fold change $\geq 2$ were marked significantly different in DSI (UP vs. SP) group, DSC (UP vs. CP) group and $\mathrm{X}$ (CP vs. SP) groups.

\section{GO analysis}

The Blast2Go program (Conesa et al. 2005) was used to obtain GO annotations for all identified genes. To gain an understanding of the distribution of gene functions at the macro level, the WEGO online tool (Ye et al. 2006) was used to perform GO functional classification. The broad molecular function of the DEGs was analysed in terms of significantly enriched GO categories for molecular function using SEA (Du et al. 2010), in which FDR was calculated to correct the $P$ value (Pawitan et al. 2005). $P$ values $<0.05$ and FDRs $<0.05$ were defined as significant.

\section{Pathway analysis}

KEGG is a highly integrated database for systematic analysis of gene function in terms of the networks of genes and molecules (http://www.genome.jp/kegg/). KEGG pathway analysis was used to identify the significant pathways associated with the DEGs. $P$ values $<0.05$ and FDRs $<0.05$ were defined as significant (Kanehisa et al. 2008; Yi et al. 2006).

\section{qRT-PCR analysis}

TransScript One-Step gDNA Removal and cDNA Synthesis SuperMix kit (TransGen, Beijing, China) was used to synthesize the first strand cDNA, according to the manufacturer's instructions. TransStart Tip Green qPCR SuperMix (TransGen, Beijing, China) and the Roche LightCycler 480IIPCR system (Roche Diagnostics GmbH, USA) were used to perform the qRT-PCR. After a total volume of $20 \mu \mathrm{l}$ reaction mixture was prepared, amplification was carried out with the following cycling parameters: $95{ }^{\circ} \mathrm{C}$ for $30 \mathrm{~s}$, followed by 45 cycles of $95^{\circ} \mathrm{C}$ for $5 \mathrm{~s}$, and $60{ }^{\circ} \mathrm{C}$ for $30 \mathrm{~s}$. Three biological replicates were analysed and the expression values were normalized against actin. The specificity of the products was confirmed via agarose gel and melting curves were analysed. Analysis of the relative gene expression data was conducted using the $2^{-\Delta \Delta \mathrm{Ct}}$ method (Livak and Schmittgen 2001).

\section{Quantification of hormones}

Methyl jasmonate (JA), abscisic acid (ABA), gibberellin (GA3), indole-3-acetic acid (IAA), brassinolide (BR), and zeatin riboside $(\mathrm{ZR})$ concentrations were determined using enzyme-linked immunosorbent assay (ELISA) methods according to Yang et al. (2001). The results are presented as the mean $\pm \mathrm{SE}$ of three replicates.

\section{Results}

Pear style transcriptome assembly and differential gene expression profiles of DSI (UP vS. SP) and DSC (UP vs. CP)

A total of $65.27,68.42$, and $58.06 \mathrm{M}$ raw reads with an average Q20 over 97\% were generated from the UP, SP, and CP samples, respectively, with Illumina Solexa sequencing technology. After removing adaptors, and filtering short and low-quality reads, clean reads of $150 \mathrm{nt}$ were mapped to the reference genome. Of these highquality reads, $72.2,72.5$, and $70.2 \%$ were aligned to the pear reference database (Table 1), and a total of 26,521, 26,982, and 26,882 unigenes were obtained for the UP, SP, and $\mathrm{CP}$ samples, respectively.

To quantify the expression level of UP (control), SP (incompatible pollination), and CP (compatible pollination), RPKM values were calculated. A total of 1795 genes were differentially expressed between UP and SP (Additional file 1), including 896 downregulated and 899 upregulated genes. Similarly, 1890 genes were differentially expressed between UP and CP (Additional file 2), including 734 down-regulated and 1156 up-regulated genes. In addition, CP and SP were also compared: 520 genes were differentially expressed (Additional file 3), including 427 upregulated and 93 downregulated genes. The plots of unigenes between orange and blue revealed unigenes with both fold change and significance (Fig. 1a).

To further narrow the candidate genes related to incompatible and compatible reactions, DSI (UP vs. SP), DSC (UP vs. CP), and X (CP vs. SP) were comprehensively compared. As shown in Fig. 1b, 232 genes, including three common genes, were continuously upregulated and downregulated among UP, CP, and SP. Specifically, the three continuously downregulated genes were glycerophosphoryl diester phosphodiesterase (Pbr002321.1), alpha-galactosidase (Pbr002935.1), and epidermis-specific secreted glycoprotein EP1 (Pbr038211.1). Although some of these downregulated genes are not related to the two important KEGG pathways, they are important for research on pear SI. 
Table 1 Statistics of the reads from RNA-seq analysis and mapping results

\begin{tabular}{lllllll}
\hline Sample & Total clean reads & Single length (bp) & Total length (bp) & GC percentage & Q20 (\%) & Mapping to genome rate (\%) \\
\hline Sample UP & $64,481,698$ & 150 & $9,173,193,132$ & 46.03 & 97.64 & 72.2 \\
Sample SP & $67,636,348$ & 150 & $9,640,868,615$ & 45.92 & 97.81 & 72.5 \\
Sample CP & $57,250,730$ & 150 & $8,125,868,717$ & 45.95 & 97.71 & 70.2 \\
\hline
\end{tabular}

A
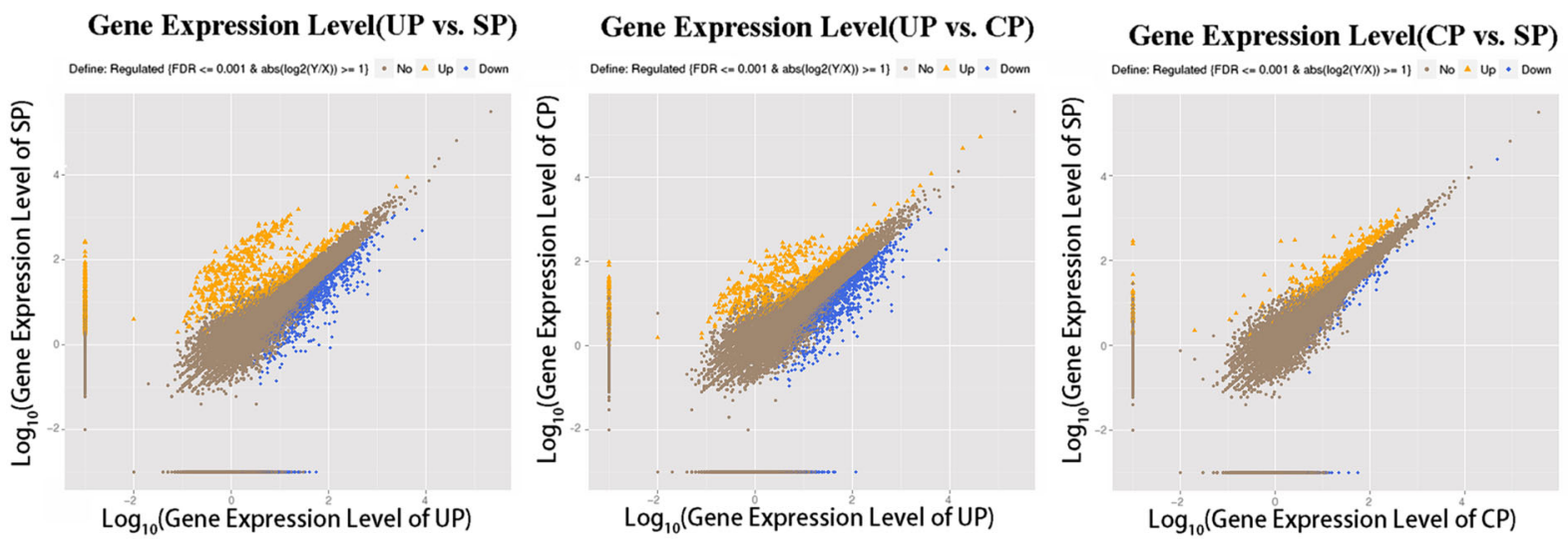

B

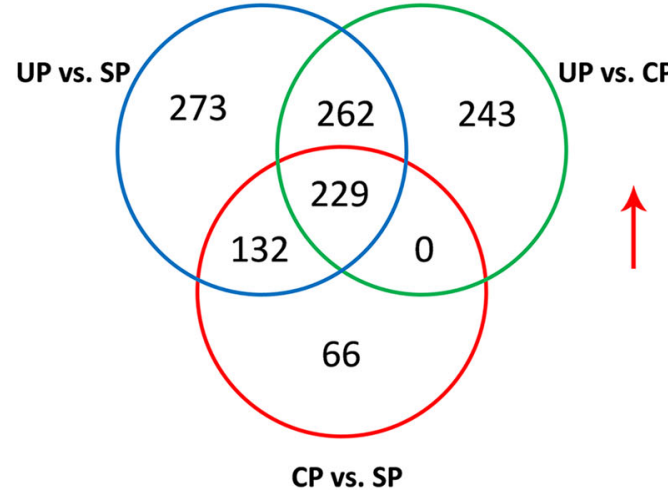

Fig. 1 Differentially expressed genes (DEGs) in DSI (UP vs. SP), DSC (UP vs. CP), and X (CP vs. SP). a Abundance of each gene was normalized as reads per kb per Millionreads (RPKM). The differentially expressed genes (DEGs) are shown in orange and blue, while

\section{Gene ontology (GO) annotation and KEGG pathway mapping of all DEGs in DSI (UP vs. SP) and DSC (UP vs. CP)}

To identify the functions of DEGs in DSI and DSC, GO analyses were performed. In total, 1890 DEGs of DSI and 1796 DEGs of DSC were assigned to GO annotations. All genes were distributed into three categories: cellular component, molecular function, and biological process. The GO terms of DEGs in DSI and DSC were categorized into the same 33 main functional groups. Cell (122 genes, $53.74 \%$ in DSI; 169 genes, $64.75 \%$ in DSC), cell part (122 genes, $53.74 \%$ in DSI; 169 genes, $64.75 \%$ in DSC), and

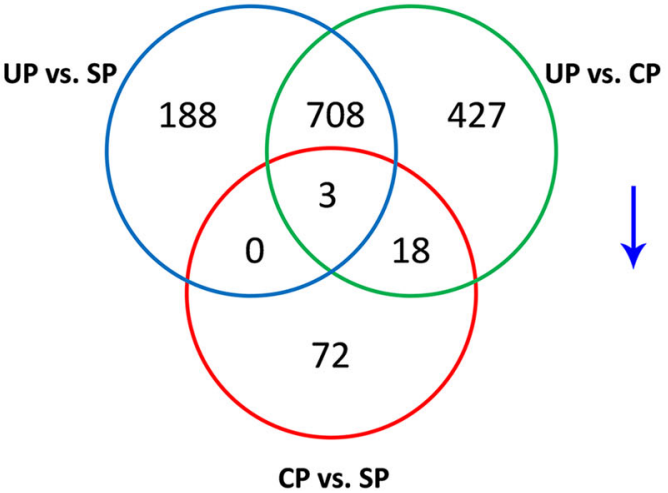

brown indicates genes that were not differentially expressed (not DEGs). b Venn diagram showing common or uniquely regulated genes among three samples. Upregulated genes are shown in the left, while downregulated genes are shown in the right (colour figure online)

membrane (103 genes, $45.37 \%$ in DSI; 93 genes, $35.24 \%$ in DSC) dominated in the cellular component category. With respect to the molecular function, genes were associated with catalytic activity (446 genes, $77.02 \%$ in DSI; 461 genes, $72.37 \%$ in DSC), binding (308 genes, $53.19 \%$ in DSI; 346 genes, $54.31 \%$ in DSC), and transporter activity (50 genes, $0.08 \%$ in DSI; 50 genes, $0.07 \%$ in DSC). Genes associated with metabolic process (339 genes, $76.17 \%$ in DSI; 385 genes, $77.00 \%$ in DSC), cellular process (220 genes, $49.43 \%$ in DSI; 244 genes, $48.80 \%$ in DSC), and localization (91 genes, $10.44 \%$ in SI; 79 genes, $15.8 \%$ in DSC) were dominant in the biological process category (Fig. 2). 
Fig. 2 Gene ontology classification of differentially expressed genes in DSI (UP vs. SP) and DSC (UP vs. CP)

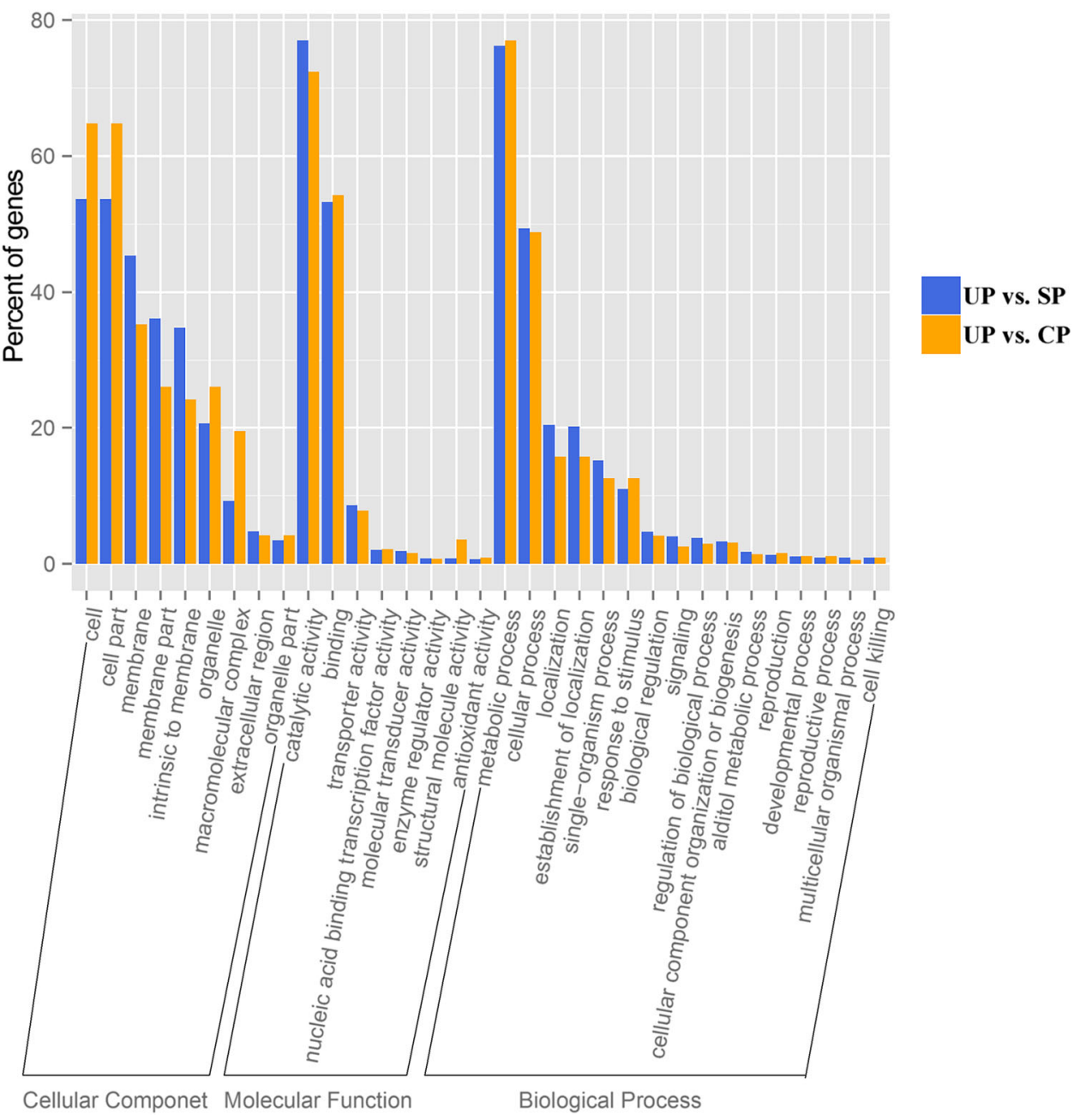

To further confirm the function of DEGs in the pathways, statistical pathway enrichment analysis of DEGs in DSI (UP vs. SP) and DSC (UP vs. CP) was performed. The DSI (UP vs. SP) and DSC (UP vs. CP) DEGs were enriched in 110 and 111 KEGG metabolic pathways, respectively. The top 20 metabolic pathways of DSI and DSC, with a $P$ value $<0.05$ and FDR $<0.05$, are shown in Fig. $3 \mathrm{a}$. Among the 110 pathways of DSI (UP vs. SP), the three containing the highest numbers of DEGs were "metabolic pathways" (301 DEGs), "plant-pathogen interaction" (116 DEGs), and "plant hormone signal transduction" (93 DEGs). Other GO terms associated with high numbers of DEGs were "starch and sucrose metabolism" (93 DEGs), "pentose and glucuronate in interconversions" (44 DEGs), and "ABC transporters" (22 DEGs). Among the 111 pathways of DSC (UP vs. CP) (Fig. 3b), those containing the highest numbers of DEGs were "metabolic pathways" (320 DEGs), "starch and sucrose metabolism" (66 DEGs)", pentose and glucuronate interconversions" (43 DEGs), and "ABC transporters" (28 DEGs). In addition, the DEGs in the "plant-pathogen interaction" and "plant hormone signal transduction" pathways were significant with respect to DSI vs. DSC, determined by a $P$ value $<0.05$ and FDR $<0.05$.

\section{Expression analysis of candidate genes}

To evaluate the validity of RNA-seq, S-RNase and 22 candidate genes that may be associated with SI reactions were selected and evaluated by qRT-PCR using genespecific primers (Fig. 4; Additional file 4; Additional file 5). These 22 genes were mainly related to transcription initiation, pathogen defence, and phytohormone signal transduction, including pectate lyase (PPL), transcription factor WRKY (WRKY), zinc finger protein (ZAT), nucleolin, transcription factor PCL (PCL), homeobox-leucine zipper protein ATHB (ATHB), brassinosteroid insensitive (BRI), serine/threonine-protein kinase (CTR), allene-oxide cyclase (AOC), jasmonate-associated MYC (MYC), 9-cis-epoxycarotenoid dioxygenase (NCED), adenylate isopentenyltransferase (AIPT), histidine kinase (AHK), GA insensitive dwarf (GID), GA sensing 
A DSI(UP vs. SP)

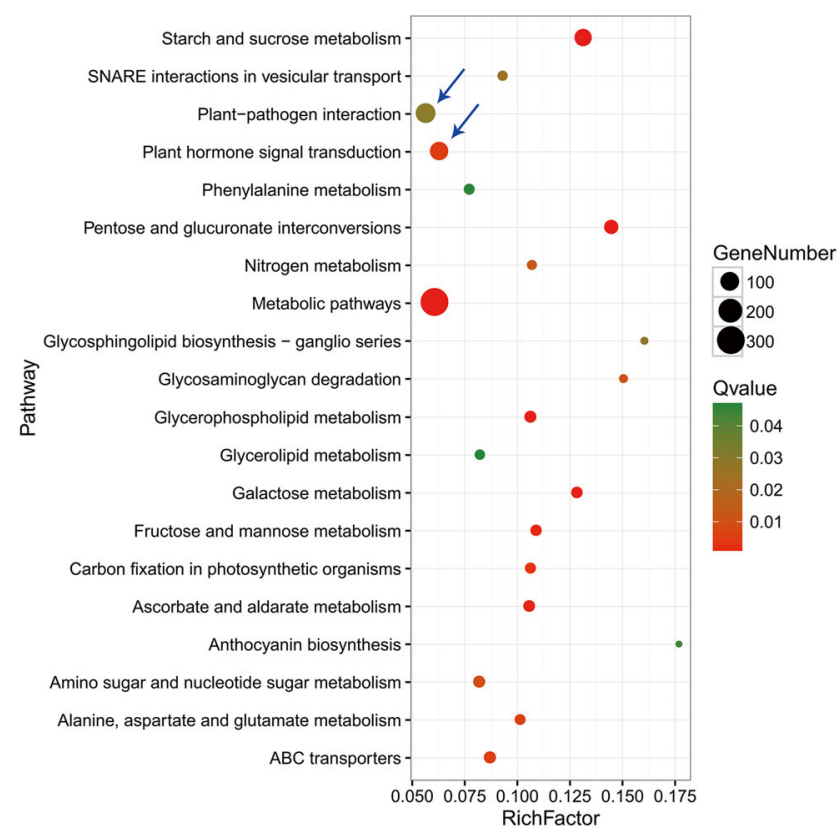

B DSC(UP vs. CP)

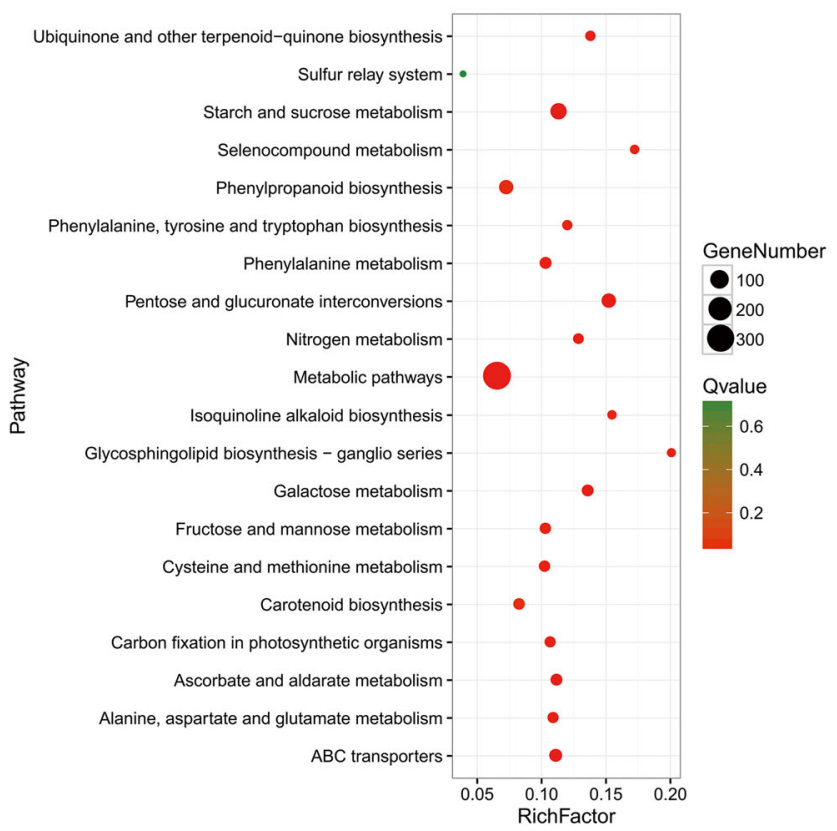

Fig. 3 Statistical scatter diagram of KEGG pathway enrichment in DSI (UP vs. SP) and DSC (UP vs. CP). a Enriched pathways in DSI (UP vs. SP). Blue arrow shows the significantly enriched pathway between DSI and DSC. b Enriched pathways in DSI (UP vs. CP) (colour figure online)

(DELLA), receptor-like serine/threonine-protein kinase (EFR), leucine-rich-repeat receptor kinases flagellin sensing (FLS), 5'-AMP-activated protein kinase (AMPK), Cyclin D (CYCD), ubiquitin-conjugating enzyme E2 $\mathrm{H}$ (UBEH), ubiquitin-conjugating enzyme E2 M (UBEM), and proteasome. Clearly, the qRT-PCR results of the selected genes showed a general agreement with the RNAseq results (Additional file 5). Of these genes, PPL, ZAT, nucleolin, BRI, CTR, MYC, AIPT, AHK, DELLA, EFR, FLS, AMPK, UBEH, UBEM, and proteasome presented significantly higher expression levels in self-pollinated (SP) styles than in unpollinated (UP) and cross-pollinated (CP) styles at $48 \mathrm{~h}$ after pollination (HAP). Other genes such as WRKY, PCL, ATHB, NCED, and CYCD showed reduced levels of expression in SP styles. However, the relative expression level of CYCD was higher in CP styles at 24 HAP (Fig. 4). These results demonstrate that defence and phytohormone signal transduction were involved in the pollination reaction.

The relative expression levels of selected genes in styles at 24 HAP were also analysed (Fig. 4) and were observed difference between 24 and 48 HAP. For example, the relative expression level of PPL was higher in DSI at 24 HAP but lower at $48 \mathrm{HAP}$. The relative expression level of CYCD decreased in unpollinated, self-pollinated, and cross-pollinated styles in a time-dependent manner. In addition, the relative expression level of PCL was not affected at 24 HAP, but it was significantly increased in UP styles compared with SP/CP styles at 48 HAP.
The S-genotype of Pyrus. bretschneideri Rehd. cv. 'Dangshansuli', which was identified by PCR using sequence-conservative primers, contains two S-RNase alleles, $S_{7}$-RNase and $S_{34}$-RNase. Unfortunately, we failed to collect the data of $S_{34}$-RNase from the RNA-seq database because of the unsuccessful assembly of $\mathrm{S}_{34}$-RNase in the sequenced pear genome. Consequently, two specific primer pairs for $S_{34}$-RNase and $S_{7}$-RNase were designed (Additional file 4 ), and qRT-PCR was performed to determine the relative expression levels at 24 and 48 HAP in UP, SP, and $\mathrm{CP}$ styles. The results showed that the relative expression levels of both $S_{7}$-RNase and $S_{34}$-RNase alleles were significantly lower in CP styles than in SP styles at 24 and 48 HAP (Fig. 4). These results indicate that compatible pollination could affect the expression level of S-RNase.

\section{Phytohormone analysis in UP, SP, and CP}

KEGG analysis revealed high enrichment in the plant hormone signal transduction pathway of DSI (UP vs. SP). To determine whether SI could stimulate phytohormone synthesis, UP, SP, and CP pear styles were collected at 24 and 48 HAP, and the concentrations of six endogenous phytohormones were measured. As shown in Fig. 5, at 24 HAP, GA3 and BR had almost identical concentrations among UP, SP, and CP styles. ZR and ABA had similar concentrations between SP and CP styles, which were higher than that in UP styles. IAA and JA-Me had similar concentrations between UP and CP styles, but which were 
PPL

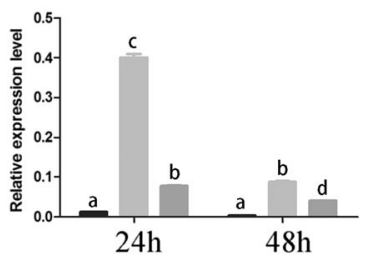

PCL

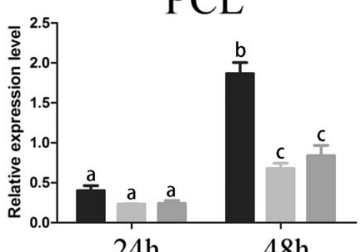

AOC

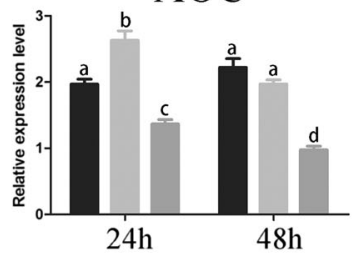

AHK

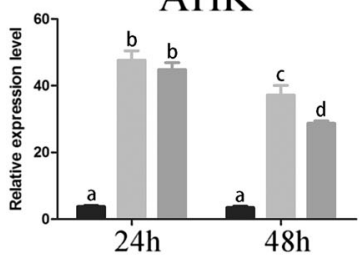

FLS

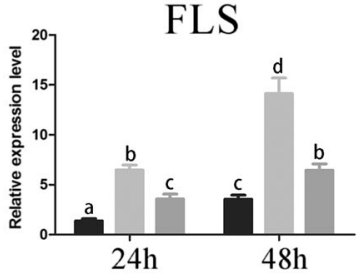

UBEM

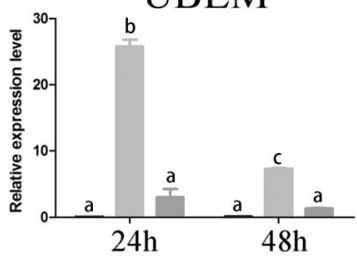

WRKY

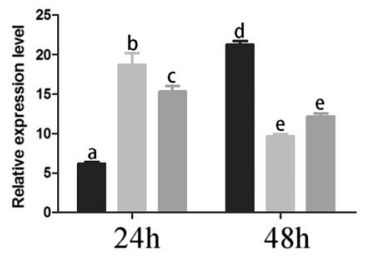

ATHB
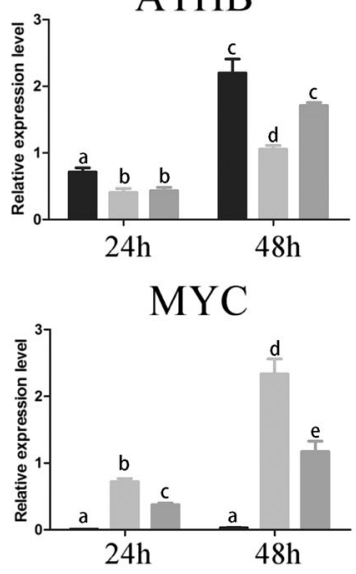

GID

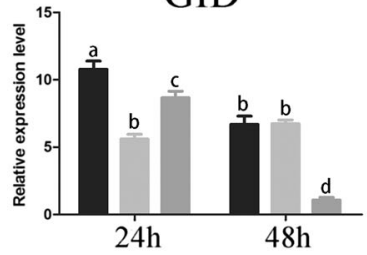

AMPK

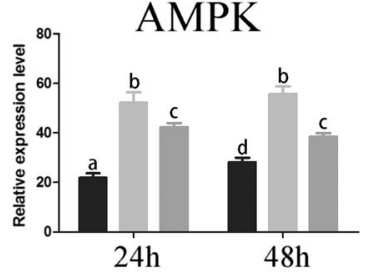

Proteasome

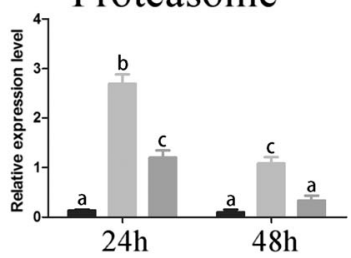

ZAT

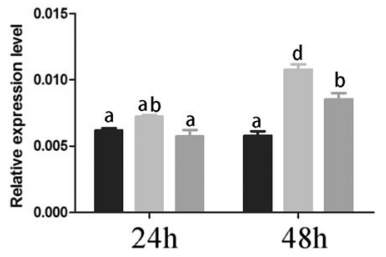

BRI

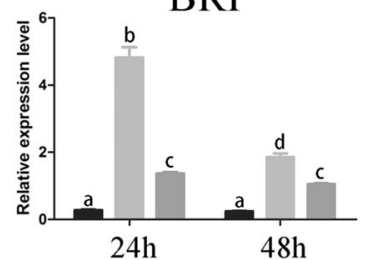

NCED

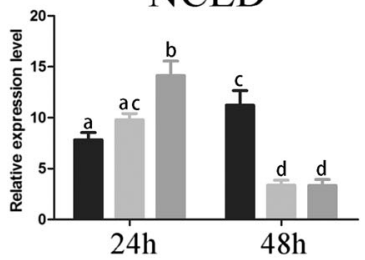

DELLA

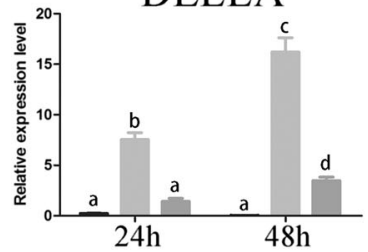

CYCD

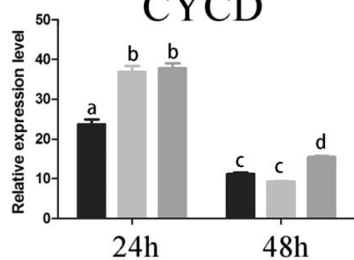

$\mathrm{S}_{7}$-RNase

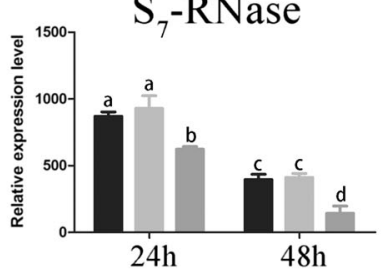

Nucleolin

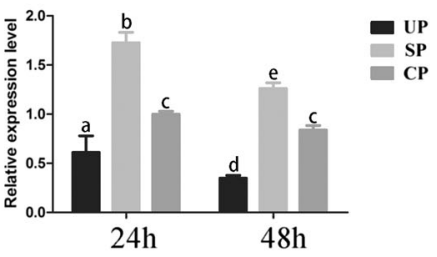

CTR

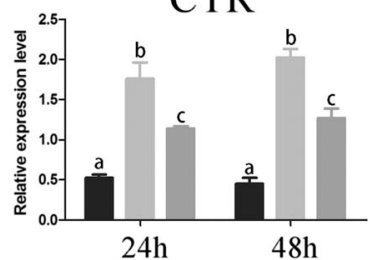

AIPT

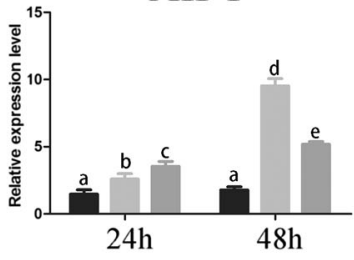

EFR

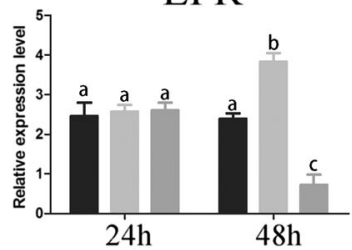

UBEH
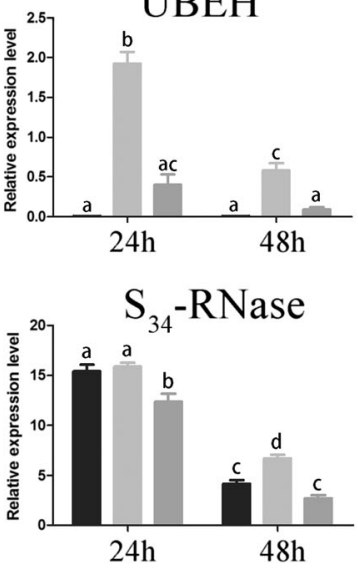

Fig. 4 Relative gene expression of selected genes in unpollinated, self-pollinated, and cross-pollinated styles after 24 and $48 \mathrm{~h}$ pollination. Relative expression level was defined as the expression level and the $X$-axis indicates hours after pollination. Bars represent the

standard deviations about the mean. Columns labeled with different letters are significantly different at $P<0.05$, Duncan's multiple range test

concentrations among UP, SP, and CP styles. GA3 had similar concentrations between UP and SP styles, which were higher than that in $\mathrm{CP}$ styles. ZR had similar concentrations between UP and CP styles, which were lower than that in SP styles. ABA had similar concentrations 

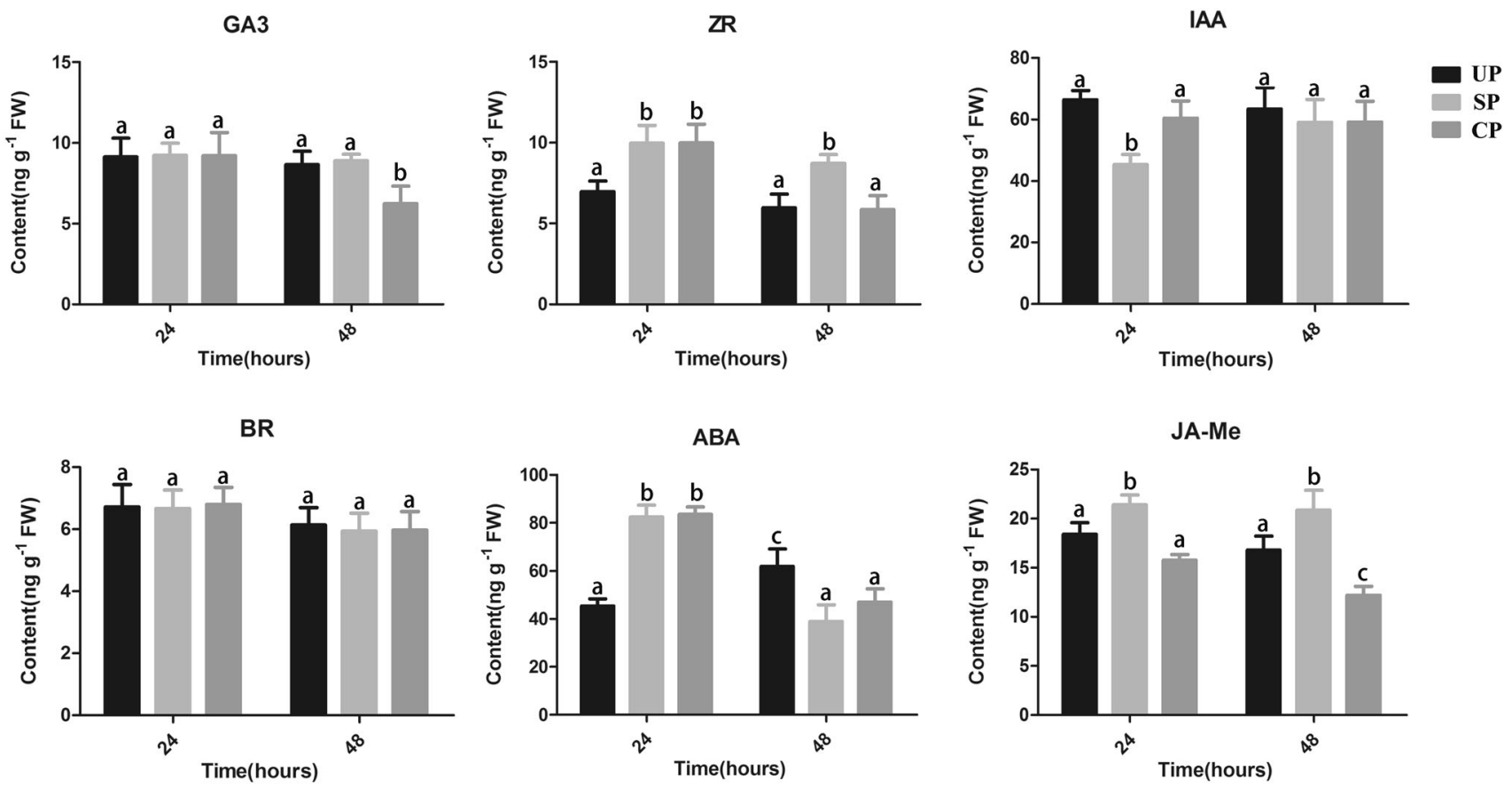

Fig. 5 Concentration of phytohormone in UP, SP, and CP after 24 and $48 \mathrm{~h}$ pollination. Bars represent the standard deviations about the mean. Columns labeled with different letters are significantly different at $P<0.05$, Duncan's multiple range test

between SP and CP styles, which were lower than that in UP styles. JA-Me had, respectively, higher and lower concentrations in SP and CP than that in UP styles. These results indicated that $\mathrm{ABA}$ was associated with pollen tube growth in SP and CP styles, ZR and JA-Me were only related to pollen tube growth in SP styles at 48 HAP. Thus, these results confirm that phytohormones play an important role in self- and cross-pollination.

\section{Expression level of S-RNase regulated by phytohormones}

RNA-seq and the phytohormone measurements demonstrate the importance of phytohormones in self- and crosspollination. In addition, phytohormones play an important role in plant growth, development, and the response to stress. To determine whether phytohormones affect the expression level of $S$-RNase alleles, the UP styles were treated with JA-Me, ABA, GA3, IAA, BR, and ZR. As shown in Fig. 6a, the expression level of $S_{34}$-RNase was markedly enhanced by JA-Me after 4 and 10 h. $S_{34}$-RNase was also induced by ABA after 10 and $24 \mathrm{~h}$. However, there was no significant difference in the expression level of $S_{34}$-RNase when treated with IAA, BR, GA3, and cytokinin (zeatin). To further determine whether $S_{7}$-RNase and $S_{34}$-RNase have the same expression patterns, specific primers were also used to analyse the relative expression level of $S_{7}$-RNase. As shown in Fig. $6 \mathrm{~b}, S_{7}$-RNase was induced by JA-Me, but the ABA treatment had no effect.
There is a possibility that $S_{7}$-RNase and $S_{34}$-RNase showed a slightly different regulatory pattern. In addition, IAA, BR, GA3, and CK could not induce the expression level of $S_{7^{-}}$ RNase, which is consistent with the findings for $S_{34}$-RNase.

To further confirm whether JA-Me and ABA could affect the expression level of $S$-RNase in other styles, 'Qingxiang' $\left(\mathrm{S}_{4} \mathrm{~S}_{7}\right)$ and 'Cuiguan' $\left(\mathrm{S}_{3} \mathrm{~S}_{5}\right)$ were selected in the second year. However, specific primers were designed between $S_{4}$-RNase and $S_{7}$-RNase in 'Yaqing' but could not be designed between $S_{3}$-RNase and $S_{5}$-RNase in 'Cuiguan' due to higher sequence identity in untranslated and translated region. As shown in Fig. 7, the relative expression levels of $S_{4}$-RNase, $S_{7}$-RNase, and $S_{3} S_{5}$-RNase were markedly enhanced by JA-Me after 4 and $10 \mathrm{~h}$ but could not be induced by ABA. Overall, we found a relationship between phytohormones and the expression level of S-RNase.

\section{Discussion}

\section{DEGs involved in SI in pear}

SI is a complex biological process that enforces outcrossing in plant populations. Exploration of the molecular mechanisms underlying the style response to self-pollen, particularly the regulation of S-RNase and the detoxification mechanism of pollen-specific S-locus $S F B$, has been important in plant genetic manipulation, genomic research, and agriculture production. RNA-seq is a rapid and efficient 
A
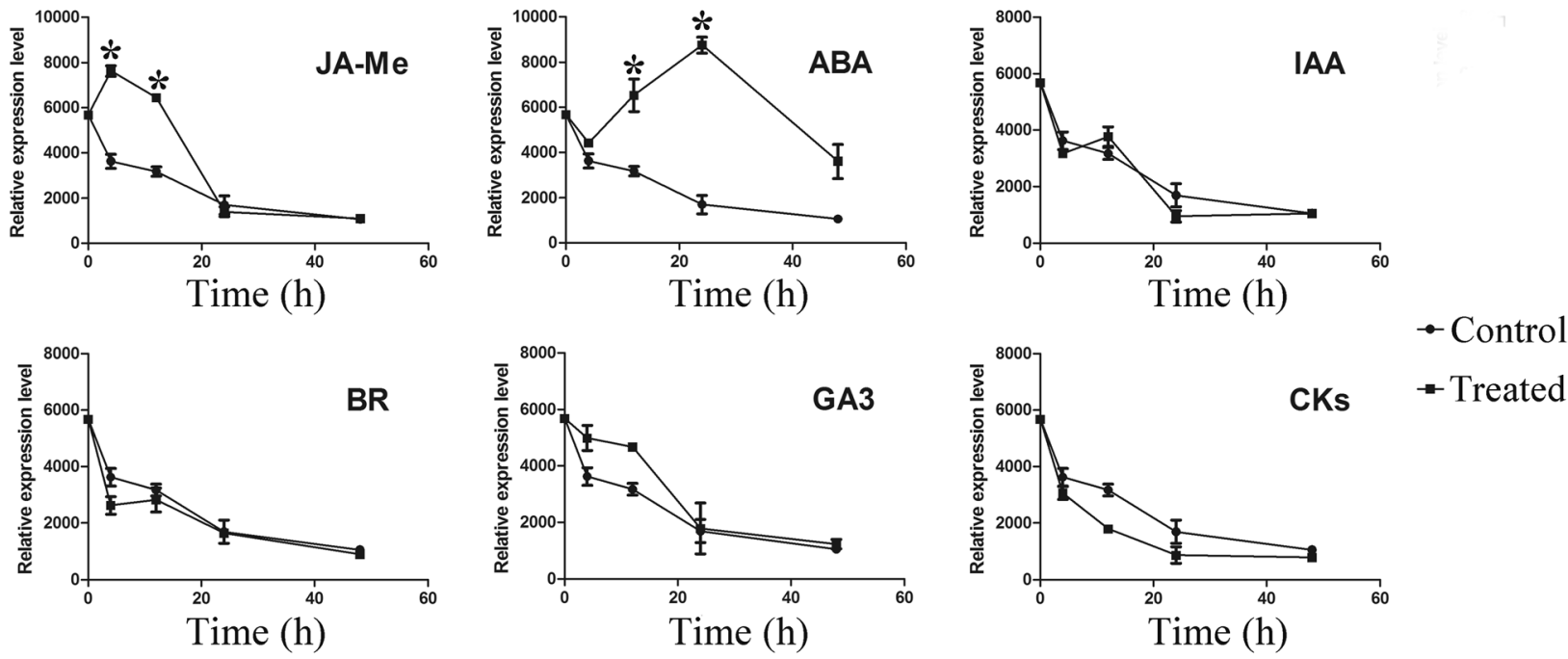

B
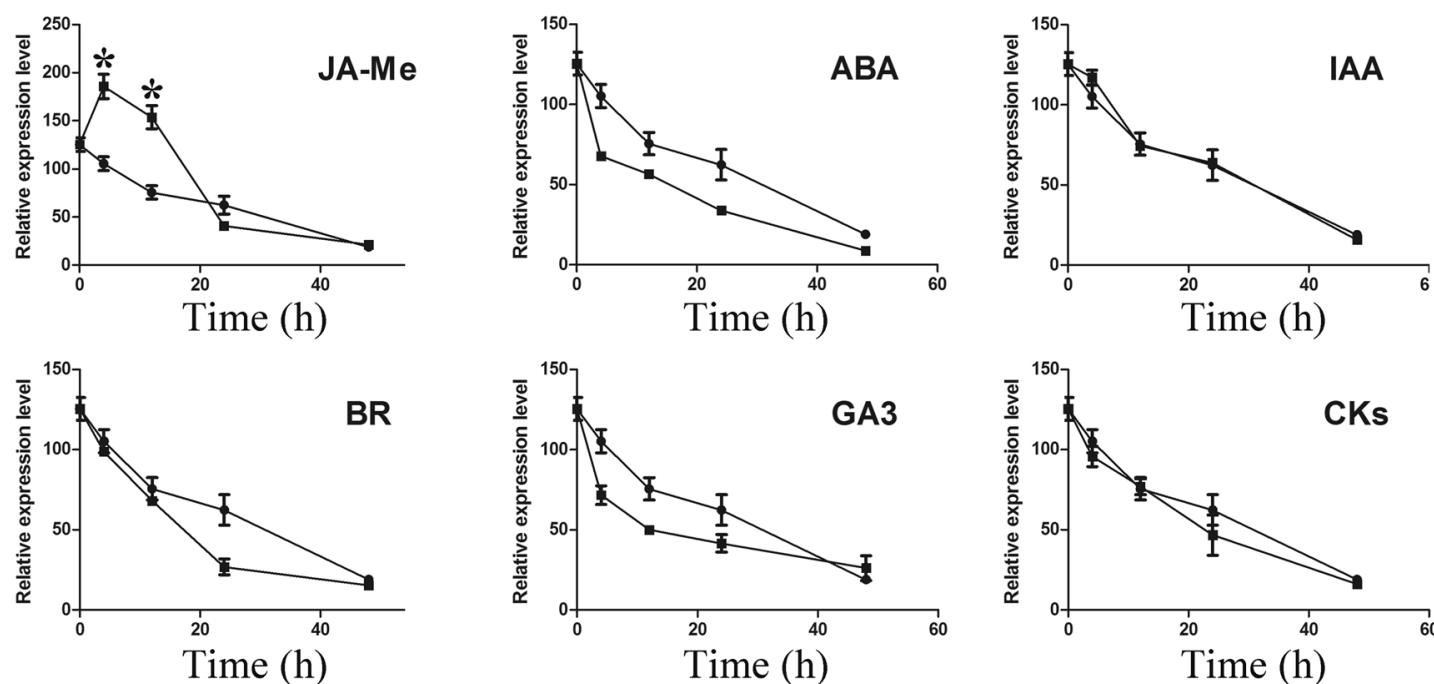

Time (h)

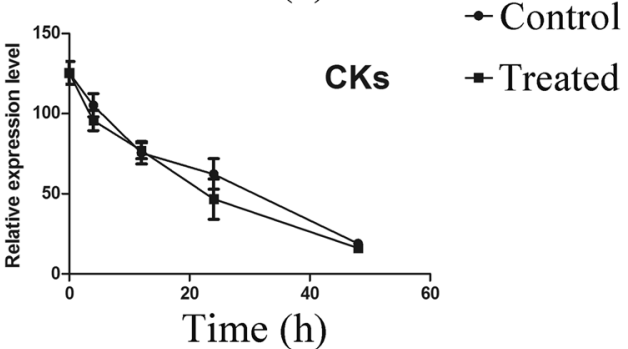

Fig. 6 Relative expression level of S-RNase in unpollinated styles with different phytohormone treatment. a Relative expression level of $S_{34}$-RNase treated with JA-Me, ABA, IAA, GA3, BR, and CKs. Bars represent the standard deviations about the mean. *Student's $t$ test

tool for obtaining a global profile of gene expression at the transcriptome level. Combined with the whole-genome database, it provides an improved bioinformatics pipeline to identify candidate genes involved in SI. In this study, 1795 DEGs of DSI, 1890 DEGs of DSC, and 520 DEGs of X (CP vs. SP) were identified in pear styles (Fig. 1); these genes provide further support that SI is regulated by multiple genes. This result is consistent with that observed for tomato styles, where 804 and 1341 DEGs were reported to be involved in incompatible and compatible pollination, respectively (Zhao et al. 2015). Moreover, 4785 and 7677 DEGs in lemon styles were associated with incompatible and compatible pollination, respectively (Zhang et al. with $P<0.05$. b Relative expression level of $S_{7}$-RNase treated with JA-Me, ABA, IAA, GA3, BR, and CKs. Bars represent the standard deviations about the mean. *Student's $t$ test with $P<0.05$

2015). In the tea SI reaction, 3182, 3575, and 3709 DEGs were identified between SP and CP styles at 24,48 , and $72 \mathrm{~h}$, respectively. The expressions of these genes changed with pollen tube growth (Zhang et al. 2016). This finding suggests that SI is a complex process that requires many aspects of gene regulation. However, when comparing CP and SP, only 520 DEGs were identified, which was approximately threefold lower than DSI and DSC in our study. This result suggests that the majority of DEGs may respond to the pollination reaction, whereas fewer DEGs respond to the selection of incompatible and compatible reactions. Thus, these data demonstrate that SI is a complex process regulated in time and space. 

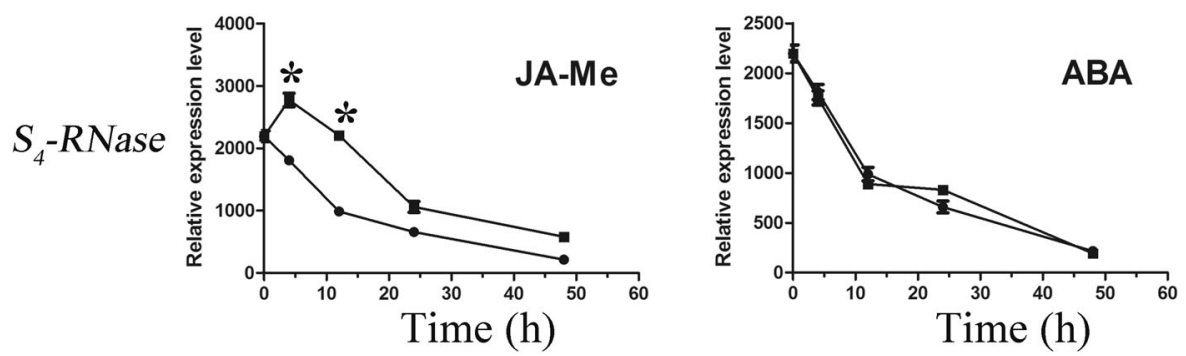

- Control

- Treated
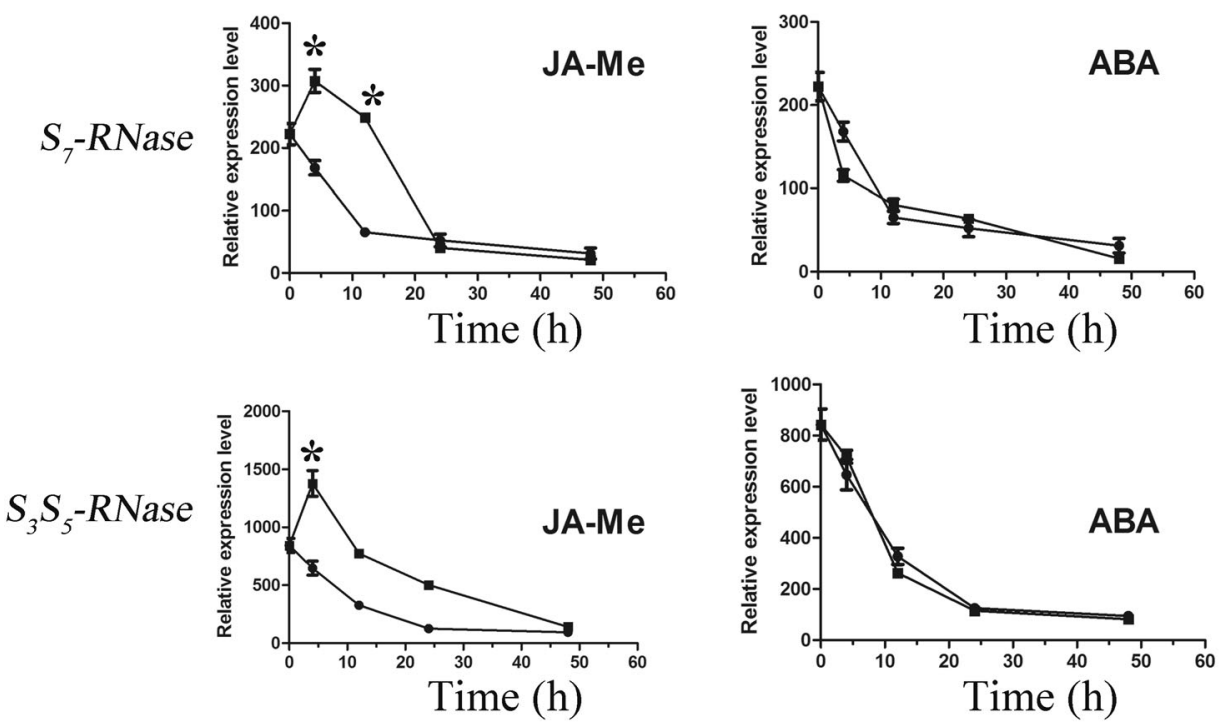

Fig. 7 Relative expression level of S-RNase in 'Qingxiang' and 'Cuiguan' styles with JA-Me and ABA treatment. 'Qingxiang' harbors S4RNase and S7-RNase; 'Cuiguan' harbors S3-RNase and S5-RNase

\section{Involvement of phytohormones in the SI reaction}

Small signalling molecules such as JA-Me are essential for plant survival in nature (Chini et al. 2007). JA-Me is a key signal in the plant response to environmental stress, such as wounding, pest attack, and pathogens (Lorenzo and Solano 2005). Our results unexpectedly indicated that the concentration of JA increased in the SP styles and decreased significantly in the CP styles (Fig. 5). In addition, JA-Me can induce the expression of S-RNase in styles. The socalled octadecanoid biosynthesis pathway is a JA biosynthesis pathway (Schaller 2001; Vick and Zimmerman 1984). Although there were no significant DEGs in DSI at 48 HAP, a key enzyme for JA biosynthesis, allene oxide cyclase (AOC, Pbr013257.1) (Li et al. 2005), was significantly downregulated in DSC but not in DSI at 48 HAP that was upregulated in DSI at 24 HAP. MYC, a basic helix-loop-helix leucine zipper transcription factor localized in the nucleus, is induced by JA. JA can induce two groups of genes: genes involved in defence responses against pathogens that are repressed by MYC and genes involved in JA-mediated systemic responses to wounding that are activated by MYC (MYC) (Lorenzo et al. 2004). We observed that the expression level of jasmonate- associated MYC (MYC, Pbr029553.1) was lower in DSC compared to that in DSI at 24 and 48 HAP, which is consistent with the reduced concentration of JA-Me in DSC. In addition, the expression level of MYC in DSI and DSC was higher at 48 HAP than at 24 HAP (Fig. 4). However, although the trend of the expression level of MYC was not consistent with a group of genes involved in defence response against pathogens in Arabidopsis, it was related to another group involved in JA-mediated systemic responses to wounding. This shows that signal transduction of MYC in pear may be different from that in Arabidopsis. Combined with exogenous JA treatment, we believe that the reduced expression level of S-RNase is related to the JA-signalling cascade.

$\mathrm{ABA}$ is an important regulator of several physiological and plant defence processes such as the response to heat and drought stresses: similar to JA, ABA can also be induced by water stress (Jubany-Marí et al. 2010; Planchet et al. 2011). The carotenoid biosynthesis pathway was enriched in DSI and DSC. Endogenous ABA biosynthesis is regulated by 9-cis-epoxycarotenoid dioxygenase (NCED, Pbr009089.1), which was significantly downregulated in DSI and DSC. Phytohormone analysis showed that the ABA concentration increased at 24 HAP but decreased at 
48 HAP in DSI and DSC (Fig. 5). This result is consistent with the ABA synthesis pathway in 48 HAP. PP2C phosphatases, i.e., negative regulators of the ABA signal, can be induced in response to $\mathrm{ABA}$, salt, and drought treatments (Chen et al. 2015). In the current study, the expression levels of PP2C phosphatases (Pbr033165.1, Pbr041497.1, Pbr022745.1, Pbr013576.1, Pbr015521.1, Pbr018965.1, Pbr032029.1, Pbr042867.1, Pbr013022.1, Pbr020818.1, and Pbr011405.1) were enriched in self- and cross-pollination. However, we could not confirm which PP2C phosphatases were the major participant in self-pollination. This is an interesting topic for our further research on pear SI. Taken together, these results suggest that the ABAsignalling cascade participates in the style response to pollen.

Cytokinin (CK) can regulate cell division, leaf senescence, nutrient mobilization, apical dominance, and seed germination (Hwang et al. 2012). In Petunia hybrid plants, the inhibition of pollen tube growth coincided with a fivefold increase in the CK content in the style (Kovaleva and Zakharova 2004). In the present study, the concentration of ZR increased in SP/CP styles after $24 \mathrm{~h}$ and decreased $48 \mathrm{~h}$ after cross-pollination. This result suggests that the signalling cascade of cytokinin changed when the pollen tube reached the base of the style, and the signalling cascade of cytokinin decreased at $48 \mathrm{~h}$ after cross-pollination. Adenylate isopentenyltransferase (AIPT, Pbr038052.1) is a key enzyme involved in zeatin biosynthesis. In addition, histidine kinase (AHK, Pbr026909.1) is a cytokinin receptor involved in cytokinin signal transduction. In our study, these two genes were significantly upregulated in DSI but not in DSC. This is consistent with the change in the concentration of $\mathrm{ZR}$ in the styles, suggesting that cytokinin participates in pollen discrimination but not in the synthesis of S-RNase in pear. A recent study revealed that $\mathrm{CK}$ is involved in a number of plant-pathogen interactions (Naseem et al. 2012). It was speculated that cytokinin participated in reducing the plant-pathogen interactions in the CP styles. Based on these findings, it can be concluded that $\mathrm{CK}$ is also involved in the SI reaction in pear.

The DELLA protein acts as a plant growth repressor, and GID1 is a GA receptor. GAs trigger the formation of a GA-GID1-DELLA complex, which forms ubiquitinated DELLA. Finally, DELLA is degraded by $26 \mathrm{~S}$ protein and the plant growth repressor is dismissed. In the present study, the expression level of GA sensing (DELLA, Pbr035217.1) was enhanced in SP pear styles, which is consistent with self-pollinated lemon (Zhang et al. 2015). This finding suggests that a universal phenomenon exists in the function of DELLA in GSI. In addition, the expression level of GA insensitive dwarf (GID, Pbr029089.1) was lower in $\mathrm{CP}$ styles which are consistent with the reduced concentration of GA in CP styles. Taken together, it can be concluded that GA is also involved in the SI reaction in pear.

Thus, the results suggest that phytohormones are involved in the SI reaction in pear.

\section{Plant-pathogen signal in the SI reaction}

The interaction between pollen and style shares similarities with bacterial infection in terms of biological responses (Van Doorn and Woltering 2008) and some pathogen defence genes, such as leucine-rich-repeat receptor kinases flagellin sensing (FLS) and LRR receptor-like serine/threonine-protein kinase (EFR) (Tintor et al. 2013). The increased expression of FLS (Pbr037634.1) and EFR (Pbr029003.1) in DSI suggests the activation of pathogen defence, which is similar to the SI reaction in that defence is initiated by styles to inhibit growth. By contrast, the expression levels of FLS (Pbr037634.1) and EFR (Pbr029003.1) decreased in DSC compared with DSI. Styles can recognize self-/cross-pollen, just as plants can discriminate between wounding and pathogen invasion. It is possible that the change in the expression level of $\mathrm{S}$-RNase is a signal in pollen recognition. These results suggest that some kind of signal is required to reduce the S-RNase level (Liu et al. 2009).

The pathogen invasion response was highly enriched in the "plant-pathogen interaction" KEGG pathway ( $P$ value $<0.05)$, which involved PAMP-triggered immunity, a hypersensitive response, phytoalexin accumulation, and other responses to fungus in DSI but not in DSC (Fig. 3a). In tomato, although pollination induces the same KEGG pathway, there was no significant difference between compatible and incompatible pollination at 24 HAP (Zhao et al. 2015). In addition, it was speculated that SI has evolved from defence against pathogens (Kao and McCubbin 1996), and the defence reaction was weak at 24 HAP because of the expression levels of FLS and EFR mentioned above. It is possible that the pollination time leads to a different KEGG pathway enrichment. We assume that incompatible and compatible pollen tubes were recognized as foreign incursions at the beginning of pollination. With the continuous pollen-style communication, the style recognizes incompatible pollen as a foreign incursion and triggers the defence reaction; however, compatible pollen will be accepted and the defence reaction will decrease. We concluded that plant-pathogen interactions participate in the pear SI reaction and in the pollen discrimination in pear.

\section{Style S-RNase biosynthesis}

$\mathrm{S}-\mathrm{RNase}$ is a major protein that is involved pollen recognition and is expressed mainly in styles. A direct method to 
inhibit SI in pear is to knock out the S-RNase gene. Because of a longer period of early development and late flowering stage and genetic diversity (Wu et al. 2013), it is difficult and time-consuming to produce self-compatible transgenic breeds. Another indirect method to inhibit SI is to regulate the S-RNase expression or translation levels in real time. During the young bud stages, the SI reaction is weak in some GSI species (Gradziel and Robinson 1989; Qin et al. 2006). Although young bud pollinations are frequently used to overcome SI in certain species (Gradziel and Robinson 1989), they are not widely used in agriculture production largely due to petal detachment. In the present study, the reduced expression level of S-RNase in compatible pollination was consistent with the reduced concentration of JA-Me in the CP styles. In addition, S-RNase in the UP styles decreased at the translation level, and the expression level decreased in a time-dependent manner after flowering (Fig. 6). It seems that a compatible pollen tube can reduce $\mathrm{S}-\mathrm{RNase}$, and a mechanism exists in the style that degrades S-RNase at the translation level, reducing the expression level in cross-pollination at post flowering. Therefore, further studies are required to design methods to accelerate this process, which would help in overcoming SI in agriculture production.

S-like RNases can be induced in response to mechanical wounding and phytohormone treatment (Groß et al. 2004; Rogers and Rogers 1999; Hillwig et al. 2008). In the present study, JA-Me induced the expression of S-RNase, but the concentration of JA-Me in self-pollinated styles was not significantly higher than that in UP styles. In addition, the concentration of JA-Me was lower in CP styles, which could be a contrary evidence to prove that a relationship exists between JA-Me and the expression of S-RNase. We suggest that JA-Me is involved in pear SI via the induction of the expression of S-RNase. However, more work is required to identify the downstream factors. ABA could only induce $S_{34}$-RNase, and the effect of ABA was delayed (Fig. 6). ABA triggered JA accumulation in Salvia miltiorrhiza hairy roots (Yang et al. 2012), but further studies are required to confirm whether it can trigger JA biosynthesis in pear styles. The ABA signals appear to share different mechanisms with JA in regulating the expression of S-RNase.

\section{Potential interference of the DEG results}

Because it is difficult to remove the pollen tube from SP to $\mathrm{CP}$ styles, we selected styles containing pollen. Hence, there is the possibility of pollen interference in the few DEGs, or genes were not significantly expressed in the styles. Nevertheless, the quantity of pollen in the styles was relatively lower than the total quantity of the styles, and research (Zhang et al. 2015, 2016; Zhao et al. 2015) on SI in other species confirmed our finding that styles containing pollen can be used to analyse the SI reaction in styles. In addition, the expression level of some organ-specific genes such as style-specific S-RNase was not affected. Thus, although there was a potential interference of the DEG results, this method can help in narrowing the range of candidate genes in further research on the SI reaction.

In summary, a comprehensive transcriptome-based characterization of the DEGs involved in self- and crosspollination was performed in pear styles. Candidate genes related to incompatibility and compatibility reactions were identified. Two major KEGG pathways, "plant hormone signal transduction" and "plant-pathogen interaction", were emphasized in pear SI reaction. JA-Me and ABA may enhance the expression level of S-RNase and pollination could affect the synthesis of JA-Me and ABA in pear styles. It appeared that there is relationship between selfrejection, plant hormones, and pathogen defence in pear SI. Therefore, the question of how phytohormones affect the synthesis of S-RNase, how pathogen defence participates in pear SI, can be examined further.

Author contribution statement DS carried out the experiments, data analysis, and preparation of figures, and drafted the manuscript. CT and RW participated in the experiments and data analysis. CG participated in the data analysis and preparation of figures, and contributed with consultation. XW, SH, and JJ contributed to sample collection and data analysis. SZ managed and designed the research and experiments.

Acknowledgements This work was supported by the National Hightech R\&D Program of China (863 Program, 2013AA102606-02), the Ph.D. Programs Foundation of Ministry of Education of China (20130097130004), Jiangsu Province, Science and Technology Support Program, China (BE2014400), China Agriculture Research System (CARS-29), and National Natural Science Foundation of China (31440074).

\section{Compliance with ethical standards}

Conflict of interest The authors have no conflicts of interest declared.

Open Access This article is distributed under the terms of the Creative Commons Attribution 4.0 International License (http://crea tivecommons.org/licenses/by/4.0/), which permits unrestricted use, distribution, and reproduction in any medium, provided you give appropriate credit to the original author(s) and the source, provide a link to the Creative Commons license, and indicate if changes were made.

\section{References}

Anders S, Pyl PT, Huber W (2014) HTSeq-a Python framework to work with high-throughput sequencing data. Bioinformatics 31:166-169 
Audic S, Claverie J-M (1997) The significance of digital gene expression profiles. Genome Res 7:986-995

Chen J, Zhang D, Zhang C, Xia X, Yin W, Tian Q (2015) A putative $\mathrm{PP} 2 \mathrm{C}$-encoding gene negatively regulates ABA signaling in Populus euphratica. PLoS One 10:e139466

Chini A, Fonseca S, Fernandez G, Adie B, Chico J, Lorenzo O, Garcia-Casado G, Lopez-Vidriero I, Lozano F, Ponce M (2007) The JAZ family of repressors is the missing link in jasmonate signalling. Nature 448:666-671

Conesa A, Götz S, García-Gómez JM, Terol J, Talón M, Robles M (2005) Blast2GO: a universal tool for annotation, visualization and analysis in functional genomics research. Bioinformatics 21:3674-3676

de Nettancourt D (1997) Incompatibility in angiosperms. Sex Plant Reprod 10:185-199

Du Z, Zhou X, Ling Y, Zhang ZH, Su Z (2010) agriGO: a GO analysis toolkit for the agricultural community. Nucleic Acids Res 38:W64-W70

Eaves DJ, Flores-Ortiz C, Haque T, Lin Z, Teng N, Franklin-Tong VE (2014) Self-incompatibility in Papaver: advances in integrating the signalling network. Biochem Soc Trans 42:370-376

Gradziel TM, Robinson RW (1989) Breakdown of self-incompatibility during pistil development in Lycopersicon peruvianum by modified bud pollination. Sex Plant Reprod 2:38-42

Groß N, Wasternack C, Köck M (2004) Wound-induced RNaseLE expression is jasmonate and systemin independent and occurs only locally in tomato (Lycopersicon esculentum cv. Lukullus). Phytochemistry 65:1343-1350

Hancock CN, Kondo K, Beecher B, McClure B (2003) The S-locus and unilateral incompatibility. Philos Trans $\mathrm{R}$ Soc Lond 358: $1133-1140$

Hillwig MS, Lebrasseur ND, Green PJ, Macintosh GC (2008) Impact of transcriptional, ABA-dependent, and ABA-independent pathways on wounding regulation of RNS1 expression. Mol Genet Genomics 280:249-261

Hiratsuka S, Zhang SL, Nakagawa E, Kawai Y (2001) Selective inhibition of the growth of incompatible pollen tubes by S-protein in the Japanese pear. Sex Plant Reprod 13:209-215

Huang S, Lee H-S, Karunanandaa B, T-h Kao (1994) Ribonuclease activity of Petunia inflata $\mathrm{S}$ proteins is essential for rejection of self-pollen. Plant Cell 6:1021-1028

Hwang I, Sheen J, Müller B (2012) Cytokinin signaling networks. Annu Rev Plant Biol 63:353-380

Indriolo E, Safavian D, Goring DR (2014) The ARC1 E3 ligase promotes two different self-pollen avoidance traits in Arabidopsis. Plant Cell 26:1525-1543

Jubany-Marí T, Prinsen E, Munné-Bosch S, Alegre L (2010) The timing of methyl jasmonate, hydrogen peroxide and ascorbate accumulation during water deficit and subsequent recovery in the Mediterranean shrub Cistus albidus L. Environ Exp Bot 69:47-55

Kanehisa M, Araki M, Goto S, Hattori M, Hirakawa M, Itoh M, Katayama T, Kawashima S, Okuda S, Tokimatsu T (2008) KEGG for linking genomes to life and the environment. Nucleic Acids Res 36:D480-D484

Kao T-H, McCubbin AG (1996) How flowering plants discriminate between self and non-self pollen to prevent inbreeding. PNAS 93:12059-12065

Kovaleva LV, Zakharova EV (2004) Gametophyte-sporophyte interactions in the pollen-pistil system: 4 . the hormonal status and the mechanism of self-incompatibility. Russ J Plant Physiol $51: 446-451$

Li CY, Schilmiller AL, Liu GH, Lee GI, Jayanty S, Sageman C, Vrebalov J, Giovannoni JJ, Yagi K, Kobayashi Y, Howe GA (2005) Role of $\beta$-oxidation in jasmonate biosynthesis and systemic wound signaling in tomato. Plant Cell 17:971-986
Liu ZQ, Xu GH, Zhang SL (2007) Pyrus pyrifolia stylar S-RNase induces alterations in the actin cytoskeleton in self-pollen and tubes in vitro. Protoplasma 232:61-67

Liu B, Morse D, Cappadocia M (2009) Compatible pollinations in Solanum chacoense decrease both S-RNase and S-RNase mRNA. PLoS One 4:e5774

Liu W, Fan JB, Li JH, Song YZ, Li Q, Ye Zhang, Xue YB (2014) SCF SLF-mediated cytosolic degradation of S-RNase is required for cross-pollen compatibility in S-RNase-based self-incompatibility in Petunia hybrida. Front Genet 5:228

Livak KJ, Schmittgen TD (2001) Analysis of relative gene expression data using real-time quantitative PCR and the $2^{-\Delta \Delta \mathrm{Ct}}$ method. Methods 25:402-408

Lorenzo O, Solano R (2005) Molecular players regulating the jasmonate signalling network. Curr Opin Plant Biol 8:532-540

Lorenzo O, Chico JM, Sánchez-Serrano JJ, Solano R (2004) Jasmonate-insensitive1 encodes a MYC transcription factor essential to discriminate between different jasmonate-regulated defense responses in Arabidopsis. Plant Cell 16:1938-1950

McClure B (2004) S-RNase and SLF determine S-haplotype-specific pollen recognition and rejection. Plant Cell 16:2840-2847

Mortazavi A, Williams BA, McCue K, Schaeffer L, Wold B (2008) Mapping and quantifying mammalian transcriptomes by RNASeq. Nat Methods 5:621-628

Naseem M, Philippi N, Hussain A, Wangorsch G, Ahmed N, Dandekar T (2012) Integrated systems view on networking by hormones in Arabidopsis immunity reveals multiple crosstalk for cytokinin. Plant Cell 24:1793-1814

Nasrallah JB, Nasrallah ME (2014) Robust self-incompatibility in the absence of a functional ARC1 gene in Arabidopsis thaliana. Plant Cell 26:3838-3841

Pawitan Y, Michiels S, Koscielny S, Gusnanto A, Ploner A (2005) False discovery rate, sensitivity and sample size for microarray studies. Bioinformatics 21:3017-3024

Planchet E, Rannou O, Ricoult C, Boutet-Mercey S, Maia-Grondard A, Limami AM (2011) Nitrogen metabolism responses to water deficit act through both abscisic acid (ABA)-dependent and independent pathways in Medicago truncatula during postgermination. J Exp Bot 62:605-615

Qin X, Liu B, Soulard J, Morse D, Cappadocia M (2006) Style-bystyle analysis of two sporadic self-compatible Solanum chacoense lines supports a primary role for S-RNases in determining pollen rejection thresholds. J Exp Bot 57:2001-2013

Rogers SW, Rogers JC (1999) Cloning and characterization of a gibberellin-induced RNase expressed in barley aleurone cells. Plant Physiol 119:1457-1464

Schaller F (2001) Enzymes of the biosynthesis of octadecanoidderived signalling molecules. J Exp Bot 52:11-23

Swanson R, Edlund AF, Preuss D (2004) Species specificity in pollen-pistil interactions. Annu Rev Genet 38:793-818

Takayama S, Isogai A (2005) Self-incompatibility in plants. Annu Rev Plant Biol 56:467-489

Tintor N, Ross A, Kanehara K, Yamada K, Fan L, Kemmerling B, Nürnberger T, Tsuda K, Saijo Y (2013) Layered pattern receptor signaling via ethylene and endogenous elicitor peptides during Arabidopsis immunity to bacterial infection. PNAS 110:6211-6216

Trapnell C, Roberts A, Goff L, Pertea G, Kim D, Kelley DR, Pimentel H, Salzberg SL, Rinn JL, Pachter L (2012) Differential gene and transcript expression analysis of RNA-seq experiments with TopHat and Cufflinks. Nat Protoc 7:562-578

Tung C-W, Dwyer KG, Nasrallah ME, Nasrallah JB (2005) Genomewide identification of genes expressed in Arabidopsis pistils specifically along the path of pollen tube growth. Plant Physiol 138:977-989

Van Doorn WG, Woltering EJ (2008) Physiology and molecular biology of petal senescence. J Exp Bot 59:453-480 
Vick BA, Zimmerman DC (1984) Biosynthesis of jasmonic acid by several plant species. Plant Physiol 75:458-461

Wang CL, Xu GH, Jiang XT, Chen G, Wu J, Wu HQ, Zhang SL (2009) S-RNase triggers mitochondrial alteration and DNA degradation in the incompatible pollen tube of Pyrus pyrifolia in vitro. Plant $\mathrm{J}$ 57:220-229

Wang CL, Wu J, Xu GH, Gao YB, Chen G, Wu JY, Wu HQ, Zhang SL (2010) S-RNase disrupts tip-localized reactive oxygen species and induces nuclear DNA degradation in incompatible pollen tubes of Pyrus pyrifolia. J Cell Sci 123:4301-4309

Wilkins KA, Poulter NS, Franklin-Tong VE (2014) Taking one for the team: self-recognition and cell suicide in pollen. J Exp Bot 65:1331-1342

Wilkins KA, Bosch M, Haque T, Teng N, Poulter NS, Franklin-Tong VE (2015) Self-incompatibility-induced programmed cell death in field poppy pollen involves dramatic acidification of the incompatible pollen tube cytosol. Plant Physiol 167:766-779

Wu J, Wang ZW, Shi ZB, Zhang S, Ming R, Zhu SL, Khan MA, Tao ST (2013) The genome of the pear (Pyrus bretschneideri Rehd.). Genome Res 23:396-408

Yang JC, Zhang JH, Wang ZQ, Zhu QS, Wang W (2001) Hormonal changes in the grains of rice subjected to water stress during grain filling. Plant Physiol 127:315-323

Yang DF, Ma PD, Liang X, Wei Z, Liang ZS, Liu Y, Liu FH (2012) $\mathrm{PEG}$ and $\mathrm{ABA}$ trigger methyl jasmonate accumulation to induce the MEP pathway and increase tanshinone production in Salvia miltiorrhiza hairy roots. Physiol Plant 146:173-183

Ye J, Fang L, Zheng HK, Zhang Y, Chen J, Zhang ZJ, Wang J, Li ST, Li RQ, Bolund L, Wang J (2006) WEGO: a web tool for plotting GO annotations. Nucleic Acids Res 34:W293-W297

Yi M, Horton JD, Cohen JC, Hobbs HH, Stephens RM (2006) WholePathwayScope: a comprehensive pathway-based analysis tool for high-throughput data. BMC Bioinform 7:30

Zhang SW, Ding F, He XH, Luo C, Huang GX, Hu Y (2015) Characterization of the 'Xiangshui' lemon transcriptome by de novo assembly to discover genes associated with self-incompatibility. Mol Genet Genomics 290:365-375

Zhang CC, Wang LY, Wei K, Wu LY, Li HL, Zhang F, Cheng H, Ni DJ (2016) Transcriptome analysis reveals self-incompatibility in the tea plant (Camellia sinensis) might be under gametophytic control. BMC Genomics 17:359

Zhao PF, Zhang LD, Zhao LX (2015) Dissection of the style's response to pollination using transcriptome profiling in selfcompatible (Solanum pimpinellifolium) and self-incompatible (Solanum chilense) tomato species. BMC Plant Biol 15:119

Zhou Q, Jia J, Huang X, Yan X, Cheng L, Chen S, Li X, Peng X, Liu $\mathrm{G}$ (2014) The large-scale investigation of gene expression in Leymus chinensis stigmas provides a valuable resource for understanding the mechanisms of poaceae self-incompatibility. BMC Genomics 15:1-14 